\title{
Erratum
}

\section{Rotational excitation of $\mathrm{HC}_{3} \mathrm{~N}$ by $\mathrm{H}_{2}$ and $\mathrm{He}$ at low temperatures}

\author{
M. Wernli, L. Wiesenfeld, A. Faure, and P. Valiron
}

\begin{abstract}
Laboratoire d'Astrophysique de l'Observatoire de Grenoble, UMR 5571 CNRS/UJF, Université Joseph-Fourier, Boîte postale 53, 38041 Grenoble Cedex 09, France

e-mail: pierre.valiron@obs.ujf-grenoble.fr
\end{abstract}

A\&A 464, 1147-1154 (2007), DOI: 10.1051/0004-6361:20066112

Key words. molecular data - molecular processes - errata, addenda

Two errors related to the angular representation of the $\mathrm{HC}_{3} \mathrm{~N}-\mathrm{H}_{2}$ potential energy surface (PES) arose in the implementation of the PES fitting routine and affected the closecoupling calculations presented in Wernli et al. (2007). Fortunately, these errors affect significantly only the calculations with ortho- $\mathrm{H}_{2}\left(J_{2}=1\right)$ and the published data remain largely valid for para- $\mathrm{H}_{2}\left(J_{2}=0\right)$. In particular, all quasi-classical trajectory results are intact. We describe below the two errors and their impact on the published data and on the conclusions of the paper.

- The first error occured in the angular conventions describing the $\mathrm{H}_{2}$ relative orientation. This error caused the fitting coefficients $v_{l_{1} l_{2} l}$ with $l_{2}=2$ (Eq. (2) of the paper) to violate the conventions of the MOLSCAT scattering code.

- The second error occured in the labeling of the $v_{l_{1} l_{2} l}$ coefficients in the interface between the PES routine and MOLSCAT. This error caused $i$ ) the coefficients $v_{l_{1} l_{2} l}$ with $l_{2}=0$ to be "polluted" by the contribution of terms with the same $l_{1}, l_{2}$ but $l_{2}=2$ and $i$ ) the coefficients $v_{l_{1} l_{2} l}$ with $l_{2}=2$ to be dismissed.

As a result, the effects related to the orientation of the $\mathrm{H}_{2}$ molecule were not taken into account in our scattering treatment. These mistakes explain both why our results for ortho- $\mathrm{H}_{2}$ $\left(J_{2}=1\right)$ were very similar to those for para- $\mathrm{H}_{2}\left(J_{2}=0\right)$ and why the (closed-channel) effects induced by $J_{2}=2$ were found to be negligible. New cross sections are compared to the previous ones in the figure below. On the one hand, it can be seen that the impact of the two errors on para- $\mathrm{H}_{2}\left(J_{2}=0\right)$ cross sections is actually modest: these are modified by about $20 \%$ only (note that the dominant effect is due to the inclusion of $J_{2}=2$ in the $\mathrm{H}_{2}$ basis set, in agreement with the analytical discussion in Sect. 4.1). On the other hand, cross sections for ortho- $\mathrm{H}_{2}\left(J_{2}=1\right)$ are now different from those for para- $\mathrm{H}_{2}\left(J_{2}=0\right)$ with notably the disappearance of interferences (the even $\Delta J_{1}$ propensity rule). This latter effect reflects the importance of the $\mathrm{H}_{2}$ quadrupole and in particular of the large dipole-quadrupole interaction (as given by the coefficient $v_{123}$ ) which was set to zero in our previous calculations. Similar results are obtained at higher collisional

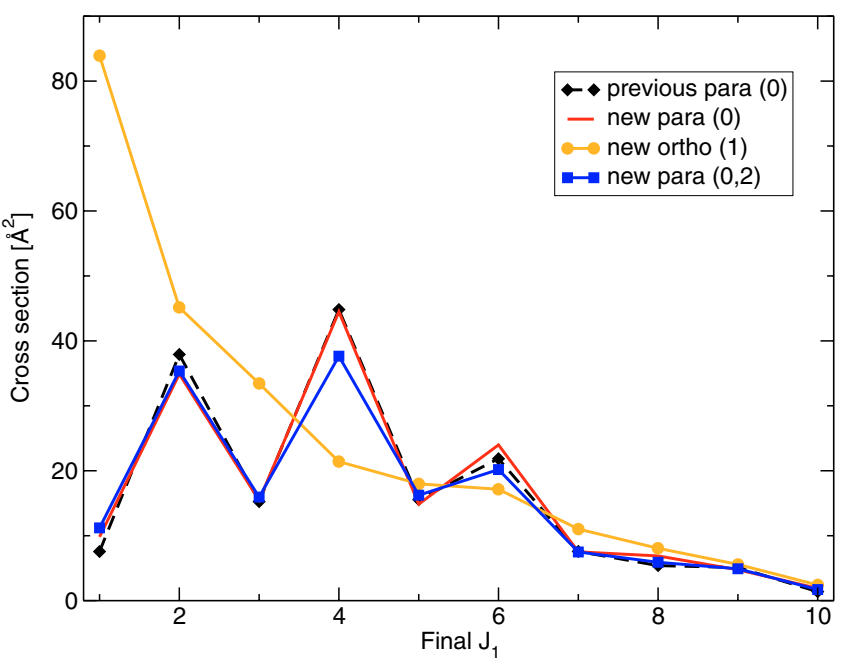

Fig. 1. Excitation cross sections from $J_{1}=0$ at a total energy of $20 \mathrm{~cm}^{-1}$. The four curves are results from the article (previous) and present calculations (new). Para and ortho stand for para- $\mathrm{H}_{2}\left(J_{2}=0\right)$ or ortho- $\mathrm{H}_{2}\left(J_{2}=1\right)$, respectively, the numbers in parentheses indicating the rotational basis used for $\mathrm{H}_{2}$.

energies, suggesting that the change in rate coefficients will be of the same order of magnitude.

The main data of the paper (Table A.1) remain valid for para$\mathrm{H}_{2}\left(J_{2}=0\right)$ (although the accuracy of the quantum rates, i.e. those for $J_{1}=0-15$, is now estimated to be about $20 \%$ only) but this data cannot be employed for ortho- $\mathrm{H}_{2}\left(J_{2}=1\right)$, which requires entirely new calculations. All other issues in the discussion remain valid.

The authors apologize for all inconveniences due to the possible use of the published rates for ortho- $\mathrm{H}_{2}\left(J_{2}=1\right)$ and thank Dr. François Lique for pointing out the errors. New rates should be available in 2008 only, due to the large CPU effort involved.

\section{References}

Wernli, M., Wiesenfeld, L., Faure, A., \& Valiron, P. 2007, A\&A, 464, 1147 